\title{
Moderate hypothermia induces protection against hypoxia/reoxygenation injury by enhancing SUMOylation in cardiomyocytes
}

\author{
JINSHENG CHEN $^{1,2^{*}}$, XIYUN BIAN ${ }^{3 *}$, YANXIA LI $^{3}$, XIAOLIN XIAO $^{3}$, YANYING YIN $^{4}$, \\ XINPING DU ${ }^{5}$, CUANCUAN WANG $^{5}$, LILI LI $^{6}$, YAOWU BAI ${ }^{1,2}$ and XIAOZHI LIU ${ }^{3}$ \\ ${ }^{1}$ North China University of Science and Technology, Tangshan, Hebei 063210; ${ }^{2}$ Department of Anesthesiology, \\ Tangshan Maternity and Child Health Care Hospital, Tangshan, Hebei 063000; ${ }^{3}$ Central Laboratory; \\ Departments of ${ }^{4}$ Neurology and ${ }^{5}$ Cardiology, The Fifth Central Hospital of Tianjin, Tianjin 300450; \\ ${ }^{6}$ Department of Bone and Soft Tissue Tumors, National Clinical Research Center for Cancer, Key Laboratory of \\ Cancer Prevention and Therapy, Tianjin Medical University Cancer Institute and Hospital, Tianjin 300060, P.R. China
}

Received November 28, 2018; Accepted March 4, 2020

DOI: $10.3892 / \mathrm{mmr} .2020 .11374$

\begin{abstract}
Moderate hypothermia plays a major role in myocardial cell death as a result of hypoxia/reoxygenation $(\mathrm{H} / \mathrm{R})$ injury. However, few studies have investigated the molecular mechanisms of hypothermic cardioprotection. Several responses to stress and other cell functions are regulated by post-translational protein modifications controlled by small ubiquitin-like modifier (SUMO). Previous studies have established that high SUMOylation of proteins potentiates the ability of cells to withstand hypoxic-ischemic stress. The level to which moderate hypothermia affects SUMOylation is not fully understood, as the functions of SUMOylation in the heart have not been studied in depth. The aim of the present study was to investigate the effect of moderate hypothermia $\left(33^{\circ} \mathrm{C}\right)$ on the protective functions of SUMOylation on myocardial cells. HL-1 and H9c2 cells were treated with the hypoxia-mimetic chemical $\mathrm{CoCl}_{2}$ and complete medium to simulate $\mathrm{H} / \mathrm{R}$ injury. Hypothermia intervention was then administered. A Cell Counting kit-8 assay was used to analyze cell viability. Mitochondrial membrane potential and the generation of reactive oxygen species (ROS) were used as functional indexes of mitochondria dysfunction. Bcl-2 and caspase-3 expression
\end{abstract}

Correspondence to: Dr Yaowu Bai, Department of Anesthesiology, Tangshan Maternity and Child Health Care Hospital, 14 Jianshe South Road, Tangshan, Hebei 063000, P.R. China

E-mail: bywyjh@163.com

Dr Xiaozhi Liu, Central Laboratory, The Fifth Central Hospital of Tianjin, 41 Zheijang Road, Tanggu Street, Tianjin 300450, P.R. China E-mail:1xz7997@126.com

${ }^{*}$ Contributed equally

Key words: hypoxia/reoxygenation, SUMOylation, moderate hypothermia, apoptosis, reactive oxygen species, mitochondrial membrane potential levels were analyzed by western blotting. The present results suggested that moderate hypothermia significantly increased SUMO1 and Bcl-2 expression levels, as well as the mitochondrial membrane potential, but significantly decreased the expression levels of caspase-3 and mitochondrial ROS. Thus, moderate hypothermia may enhance SUMOylation and attenuate myocardial H/R injury. Moreover, a combination of SUMOylation and moderate hypothermia may be a potential cardiovascular intervention.

\section{Introduction}

Cardiovascular diseases pose a threat to public health; recent estimates show that the incidence of hypertension and coronary heart disease is rising (1). Although reperfusion therapy for patients with myocardial ischemia is considered an effective treatment, reperfusion itself may induce adverse effects on the heart muscle $(2,3)$. However, suppression of apoptosis-associated pathways may improve cardiac dysfunction (4). The morphology and mitochondrial structure of HL-1 and $\mathrm{H} 9 \mathrm{c} 2$ cells are similar to primary myocardial cells in terms of biochemistry and bioenergy, and therefore these cells can be used to simulate cardiac ischemia-reperfusion injury in vitro (5). It is important to investigate myocardial protection strategies and molecular mechanisms during reperfusion.

Previous studies have shown that therapeutic hypothermia (TH) may protect the nervous system of patients with neonatal hypoxic ischemic encephalopathy or cardiac arrest $(6,7)$. Several studies have demonstrated that $\mathrm{TH}$ was effective in protecting the myocardium against hypoxia/reoxygenation $(\mathrm{H} / \mathrm{R})$-induced damage by reducing the infarct size $(8,9)$. Application of $\mathrm{TH}$ to individuals with acute myocardial infarction at temperatures $<35^{\circ} \mathrm{C}$ prior to reperfusion significantly reduced the infarct size and the release of adverse cardiac biochemical markers $(10,11)$. To maximize the cardioprotective effect of $\mathrm{TH}$, it is important to understand the protective mechanisms of hypothermia, in order to provide guidelines for managing patients with myocardial infarction. 
Post-translational modification (PTM) is crucial in regulating protein functions and stability under physiological and pathological conditions. Small ubiquitin-like modifier (SUMO)ylation is a PTM process that occurs by covalently and reversibly conjugating SUMO proteins with their target proteins (12). Previous studies have demonstrated the effects of SUMOylation on the control of several cellular functions, including cell cycle control, apoptosis, epigenetic management and transcription (13-15). Similarly, an imbalance in the SUMOylation/de-SUMOylation cycle has been implicated in several diseases (16). Previous studies have reported that multiple signaling pathways serve a role in protein SUMOylation, which mostly affect cardiac function and development $(17,18)$. SUMOylation is involved in cancer, abnormal cardiac development and cerebral ischemia (16), and promotes adaptation of the heart to pathological stress $(19,20)$, suggesting that it is a valuable target for treating cardiovascular disorders $(21,22)$. Thus, SUMOylation may have a role in controlling the response of the heart to hypoxic-ischemic stress. However, the protective roles of SUMOylation in H/R-induced myocardial cell injury have not been fully investigated. Therefore, it is imperative to examine the mechanisms of myocardial cell fate regulated by SUMOylation under physiological and pathological conditions.

Previous studies have described the link between hypothermia and SUMOylation in the brain, and indicated that hypothermia may regulate SUMOylation to provide protection to the nervous system during hypoxic ischemia $(23,24)$. However, it is unknown whether hypothermia alters SUMOylation modifications in the heart. A previous study revealed that SUMO-conjugated protein levels rose to varying degrees in organs in rats subjected to extracorporeal circulation at $18^{\circ} \mathrm{C}(25)$. Currently, there is no systematic understanding of how moderate hypothermia contributes to SUMOylation modifications in the heart. Furthermore, several aspects of cellular mechanisms responsible for the cardioprotective effects of hypothermia are relatively unknown. To further understand the mechanisms underlying hypothermia-induced cardioprotection, it is necessary to identify whether hypothermia-induced SUMOylation occurs in the heart.

The present study established an in vitro myocardial H/R model and detected the protein expression levels of SUMO1, SUMO2/3, caspase- 3 and Bcl-2. In addition, changes in reactive oxygen species (ROS) production and mitochondrial membrane potential were evaluated. The present results suggested that moderate hypothermia enhanced SUMOylation in cardiomyocytes and antagonized H/R-induced apoptosis. Thus, the present study indicated the treatment possibilities of moderate hypothermia for $\mathrm{H} / \mathrm{R}$ injury.

\section{Materials and methods}

Cell culture. HL-1 cardiomyocytes from a murine atrial tumor were provided by American Type Culture Collection (ATCC). The cells were grown at $37^{\circ} \mathrm{C}$ with $5 \% \mathrm{CO}_{2}$ in Claycomb medium (Sigma-Aldrich; Merck KGaA) containing 10\% FBS (Gibco; Thermo Fisher Scientific, Inc.), $100 \mathrm{U} / \mathrm{ml}$ penicillin, $100 \mu \mathrm{g} / \mathrm{ml}$ streptomycin, $2 \mathrm{mM} \mathrm{L}$-glutamine and $0.1 \mathrm{mM}$ norepinephrine. $\mathrm{H} 9 \mathrm{c} 2 \mathrm{embryonic}$ rat heart cells were purchased from ATCC and grown in DMEM (Gibco; Thermo Fisher
Scientific, Inc.) containing $10 \% \mathrm{FBS}$ at $37^{\circ} \mathrm{C}$ in a $5 \% \mathrm{CO}_{2}-95 \%$ air atmosphere. Cells were passaged to $80-90 \%$ confluence at ratios of $1: 3$ or $1: 4$ using trypsin with $0.05 \%$ EDTA.

Small interfering RNA (siRNA) transfection. H9c2 and HL-1 cells were cultured in complete medium for $12 \mathrm{~h}$. Transfections were performed using Lipofectamine ${ }^{\circledR} 2000$ reagent (Invitrogen; Thermo Fisher Scientific, Inc.) by incubating cells $\left(5.6 \times 10^{4}\right.$ cells/well) with the respective siRNA $(10 \mu \mathrm{M}$; developed and prepared by Shanghai GenePharma Co., Ltd.) for $48 \mathrm{~h}$. SUMO1 siRNA sequences were as follows: SUMO1 siRNA-1 (5'-TGACAACACATCTCAAGAA-3'), SUMO1 siRNA-2 (5'-GACAGGGTGTTCCAATGAA-3'), SUMO1 siRNA-3 (5'-TCTTTGAGGGTCAGAGGA7-3') and negative control siRNA, 5'-UUCUCCGACCGUGUCACGUTT-3'. In addition, SUMO1 full-length cDNA was subcloned into the mammalian expression plasmid pcDNA3.1 (Thermo Fisher Scientific, Inc.) with a Flag tag at the C-terminus. The Flag-SUMO1 plasmid and the empty vector $(0.8 \mu \mathrm{g})$ were transiently transfected into HL- 1 and H9c2 cells $\left(0.5-2 \times 10^{5}\right.$ cells/well $)$ using Lipofectamine $^{\circledR} 2000$ reagent (Invitrogen; Thermo Fisher Scientific, Inc.) according to the manufacturer's instructions. All experiments were conducted $48 \mathrm{~h}$ post-transfection.

$H / R$. H9c2 and HL-1 cells were cultured with low-glucose DMEM and subjected to chemical hypoxia using different concentrations of $\mathrm{CoCl}_{2}(50,100,300$ and $600 \mu \mathrm{M}$; HEBIO). After $12 \mathrm{~h}$ of $\mathrm{CoCl}_{2}$-induced hypoxia $\left(37^{\circ} \mathrm{C}\right)$, reoxygenation was achieved in complete medium with $10 \%$ FBS for $12 \mathrm{~h}$ $\left(37^{\circ} \mathrm{C}\right)$. Cells were subjected to $12 \mathrm{~h}$ of hypoxia and $12 \mathrm{~h}$ of reoxygenation $(H / R)$. The schematic illustration of the $H / R$ experimental protocol is shown in Fig. 1A. Cells were cultured under moderate hypothermia $\left(33^{\circ} \mathrm{C}\right)$ for $6 \mathrm{~h}$ before reoxygenation. Control cells were cultured in DMEM supplemented with $10 \% \mathrm{FBS}$ at $37^{\circ} \mathrm{C}$ in a $5 \% \mathrm{CO}_{2}-95 \%$ air atmosphere. All media were supplemented with $100 \mu \mathrm{M} / \mathrm{ml}$ penicillin/streptomycin and $2 \mathrm{mM} \mathrm{L}$-glutamine.

Moderate hypothermia protocol. The European Resuscitation Council for cardiac arrest survivors guidelines (26) were used in the present study. After $6 \mathrm{~h}$ of hypoxia, the temperature of the $\mathrm{CO}_{2}$ incubator was adjusted and maintained at $33^{\circ} \mathrm{C}$ during simulated reperfusion in the TH group of cells. During $12 \mathrm{~h}$ of reoxygenation, $\mathrm{H} 9 \mathrm{c} 2$ and HL-1 cells were incubated in DMEM containing $10 \% \mathrm{FBS}$ at $33^{\circ} \mathrm{C}$ with $5 \% \mathrm{CO}_{2}-95 \%$ air. The temperature of control group cells was maintained at $37^{\circ} \mathrm{C}$. After $24 \mathrm{~h}$ the samples were analyzed. The schematic illustration of the $H / R$ and hypothermia experimental protocol is shown in Fig. 1A.

Cell viability assay. Cell viability was examined using a Cell Counting Kit-8 (CCK-8) (Beijing Solarbio Science \& Technology Co., Ltd.) assay. H9c2 and HL-1 cells were cultured in 96 -well plates $\left(2 \times 10^{3}\right.$ cells/well) and were treated as aforementioned. Subsequently, $10 \mu 1 \mathrm{CCK}-8$ solution was added to each well and incubated at $37^{\circ} \mathrm{C}$ for $2 \mathrm{~h}$. A microplate reader (VersaMax Absorbance microplate reader; Molecular Devices) was used to analyze the absorbance at $450 \mathrm{~nm}$. To increase the reliability of measures, each experiment was performed three times. 
Determination of intracellular ROS levels. ROS was detected using the fluorescent probe 2'7'-dichlorodihydrofluorescein diacetate (DCFH-DA; Beijing Solarbio Science \& Technology Co., Ltd.). DCFH-DA has no fluorescence and can freely penetrate the cell membrane. In the presence of ROS, DCFH is oxidized into fluorescent DCF. Thus, the fluorescence intensity and the level of intracellular ROS are directly proportional. After $\mathrm{H} / \mathrm{R}, 10 \mu \mathrm{M}$ DCFH-DA was added to cells at $37^{\circ} \mathrm{C}$ for $30 \mathrm{~min}$. The cells were then washed three times with serum-free cell culture medium. The fluorescence of DCF was detected using a microplate reader (Gemini XPS microplate reader; Molecular Devices) at excitation and emission wavelengths of 488 and $525 \mathrm{~nm}$, respectively, to measure intracellular ROS levels.

Detection of changes in the mitochondrialmembrane potential. The fluorescent probe 5,5',6,6'-tetrachloro-1,1',3,3'-tetraethylbenzi-midazolylcarbocyanine iodide (JC-1; Beijing Solarbio Science \& Technology Co., Ltd.) was used to measure the mitochondrial membrane potential. After incubation at $37^{\circ} \mathrm{C}$ for $20 \mathrm{~min}$ in a cell incubator with $1 \mathrm{ml} \mathrm{JC}-1$ staining solution, the cells were washed two times using JC-1 staining buffer according to the manufacturer's instructions. Changes in laser-induced fluorescence of JC-1 were detected by confocal microscopy (Olympus Corporation).

Western blot analysis. H9c2 and HL-1 cells were washed twice with cold PBS. To avoid de-SUMOylation of proteins during sample preparation, cells were lysed for $30 \mathrm{~min}$ with $120 \mu \mathrm{l}$ ice-cold RIPA lysis buffer (Beijing Solarbio Science \& Technology Co., Ltd.) with 100:1 phenylmethanesulfonyl fluoride and 10:1 N-ethlmaleimide. The protein supernatant was collected after centrifugation at $10,000 \times \mathrm{g}$ at $4{ }^{\circ} \mathrm{C}$ for $15 \mathrm{~min}$. Protein concentration was measured using a bicinchoninic acid protein assay (Beijing Solarbio Science \& Technology Co., Ltd.). A mixture of the samples and $4 \mathrm{X}$ SDS-PAGE loading buffer was incubated for $5 \mathrm{~min}$ at $95^{\circ} \mathrm{C}$, then $40 \mu \mathrm{g} /$ lane samples underwent SDS-PAGE on $12 \%$ gels. Subsequently, proteins were transferred to a PVDF membrane at $110 \mathrm{mV}$ for $2 \mathrm{~h}$. The membrane was blocked for $1 \mathrm{~h}$ at room temperature using Tris- $\mathrm{HCl}$ containing $0.1 \%$ Tween-20 and 5\% skim milk powder (Thermo Fisher Scientific, Inc.), followed by overnight incubation at $4^{\circ} \mathrm{C}$ with anti-SUMO1 antibody (cat. no. ab11672; 1:1,000; Abcam), anti-SUMO2/3 antibody (cat. no. ab3742; 1:1,000; Abcam), anti-caspase-3 antibody (cat. no. ab13847; 1:500; Abcam), anti-Bcl-2 antibody (cat. no. ab692; 1:500; Abcam) and anti-GAPDH antibody (cat. no. ab8245; 1:1,000; Abcam). The membranes were incubated at room temperature for $1 \mathrm{~h}$ with goat anti-rabbit IgG (cat. no. 111-035-003; 1:2,000; Jackson ImmunoResearch Laboratories, Inc.) or goat anti-mouse IgG (cat. no. 115-035-003; 1:2,000; Jackson ImmunoResearch Laboratories, Inc.) as the secondary antibody. Subsequently, blotted membranes were visualized with enhanced chemiluminescence reagents (EMD Millipore). Protein bands were detected using a C-DiGit Blot Scanner (LI-COR Biosciences) and Image J software (version 1.8.0.112; National Institutes of Health) was used to normalize the data.

Statistical analysis. All HL-1 and H9c2 cell culture dishes were randomly assigned to different experimental groups $(n=5)$. Data are presented as the mean \pm SD. Statistical analysis was performed using GraphPad Prism 6.0 software (GraphPad Software, Inc.). Statistical significance was determined using a one-way ANOVA followed by Tukey's test or two-way ANOVA followed by Bonferroni's test. $\mathrm{P}<0.05$ was considered to indicate a statistically significant difference.

\section{Results}

Effects of different concentrations of $\mathrm{CoCl}_{2}$ on cell viability. CCK-8 assay was used to analyze how different concentrations of $\mathrm{CoCl}_{2}(50,100,300$ and $600 \mu \mathrm{M})$ affected the cytotoxicity of myocardial cells. The viability of HL-1 and H9c2 cells was reduced significantly with $\mathrm{CoCl}_{2}$ doses $>300 \mu \mathrm{M}$. Moreover, with 50 and $100 \mu \mathrm{M} \mathrm{CoCl}$, cell viability was decreased slightly compared with the control group (Fig. 1B and D). In addition, the cytotoxic damage was greatest at $600 \mu \mathrm{M}$. It was found that at $300 \mu \mathrm{M}$, moderate $\mathrm{TH}\left(33^{\circ} \mathrm{C}\right)$ antagonized $\mathrm{H} / \mathrm{R}$-induced cytotoxicity of cardiomyocytes (Fig. 1C and E).

Moderate TH antagonizes the decrease in mitochondrial membrane potential and ROS production triggered by $H / R$ in cardiomyocytes. In both the brain and heart, the pathological mechanisms of H/R-induced injury are homoplastic, and involve numerous processes such as mitochondrial dysfunction, intracellular acid-base disturbance and disrupted ion homeostasis $(27,28)$. During hypoxic ischemia, mitochondrial dysfunction can cause insufficient ATP production and produce excessive ROS, which impair the homeostasis of ions in cardiac cells and excitability of the cytomembrane (29). To establish whether TH has a protective function in mitochondria, the present study established a H/R model in vitro. ROS production was quantified by flow cytometry (Fig. 2A). It was revealed that after exposure to moderate $\mathrm{TH}$, the production of mitochondrial ROS was significantly reduced compared with in the $\mathrm{H} / \mathrm{R}$ group (Fig. 2B). Changes in laser-induced fluorescence of JC-1 were detected by confocal microscopy to assess the early variation of mitochondrial membrane potential (Fig. 2C). It was demonstrated that exposure of cells to moderate TH significantly increased the mitochondrial membrane potential compared with in the H/R group (Fig. 2D). Thus, the present results suggested that moderate TH improved mitochondrial functions.

Moderate TH affects protein SUMOylation after myocardial $H / R$ injury. Further experiments were performed in the present study to investigate the signaling pathways involved in protein SUMOylation. SUMOylation is an important cellular process. Previous studies have investigated the relationship between moderate hypothermia and SUMOylation, and have suggested that moderate hypothermia can trigger SUMOylation, which is an active process that regulates protein function in vitro $(23,30)$. Therefore, the aim of the present study was to determine how moderate $\mathrm{TH}$ affected protein SUMOylation in the heart. Western blotting (Fig. 3A and B) was used to detect the expression of SUMO1 and SUMO2/3 proteins, and SUMOylation of cardiomyocytes was evaluated in vitro. It was revealed that exposure of cells to $H / R$ decreased the protein expression levels of SUMO1 compared with in the control group (Fig. 3A and B). TH during reoxygenation partly reversed the reduction in SUMO1 protein expression in the 
A
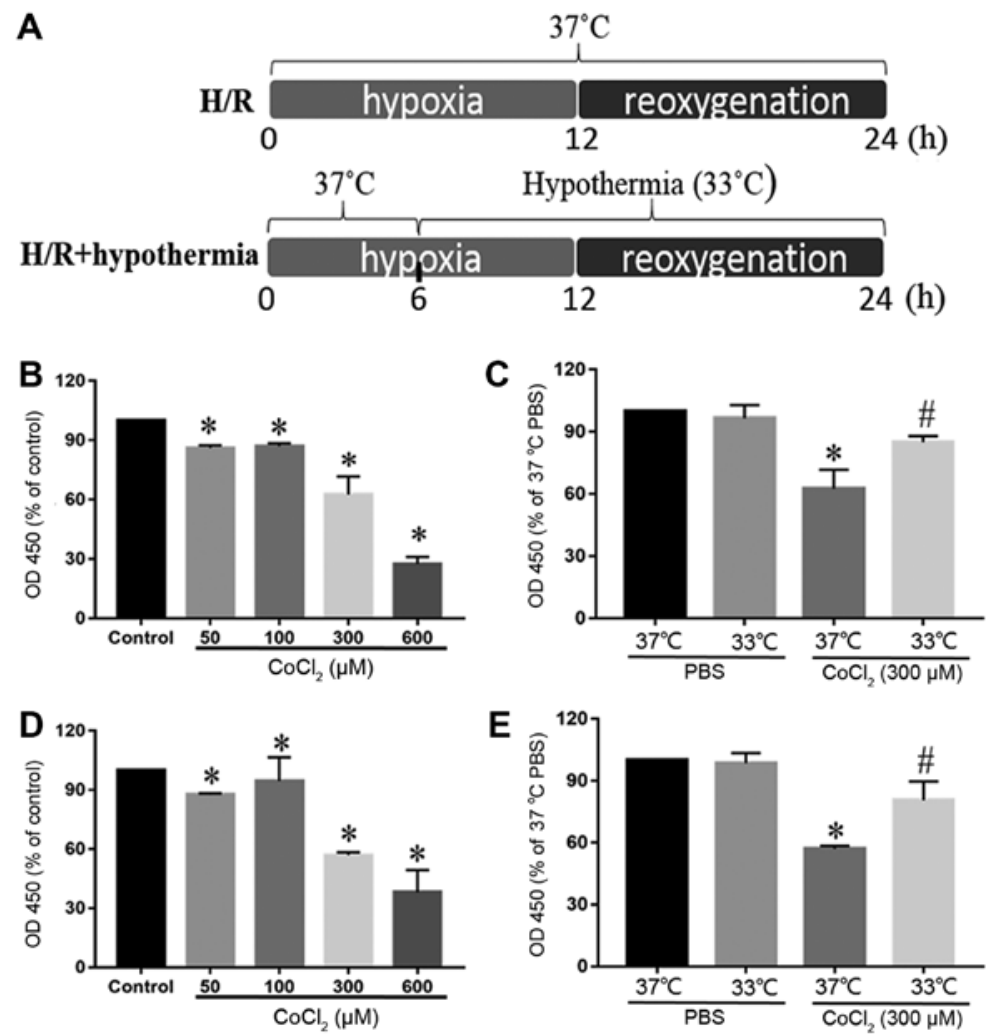

Figure 1. Effect of different concentrations of $\mathrm{CoCl}_{2}$ on cell viability. A Cell Counting Kit-8 assay was used to determine cell viability. (A) Schematic illustration of the $\mathrm{H} / \mathrm{R}$ and hypothermia experimental protocol. (B) Viability of $\mathrm{H} 9 \mathrm{c} 2$ cells subjected to $\mathrm{CoCl}_{2}$-induced hypoxia/reoxygenation. "P<0.05 vs. Control (C) Viability of $\mathrm{H} 9 \mathrm{c} 2$ cells at different temperatures. ${ }^{*} \mathrm{P}<0.05$ vs. $37^{\circ} \mathrm{C} \mathrm{PBS} ;{ }^{\sharp} \mathrm{P}<0.05$ vs. $37^{\circ} \mathrm{C} \mathrm{CoCl}{ }_{2}$. (D) Viability of HL-1 cells at different CoCl 2 concentrations. ${ }^{*} \mathrm{P}<0.05$ vs. Control. (E) Viability of HL-1 at different temperatures. ${ }^{*} \mathrm{P}<0.05$ vs. $37^{\circ} \mathrm{C} \mathrm{PBS}$; ${ }^{~} \mathrm{P}<0.05$ vs. $37^{\circ} \mathrm{C} \mathrm{CoCl}{ }_{2}$. Data are expressed as means $\pm \mathrm{SE}(\mathrm{n}=5$ ).
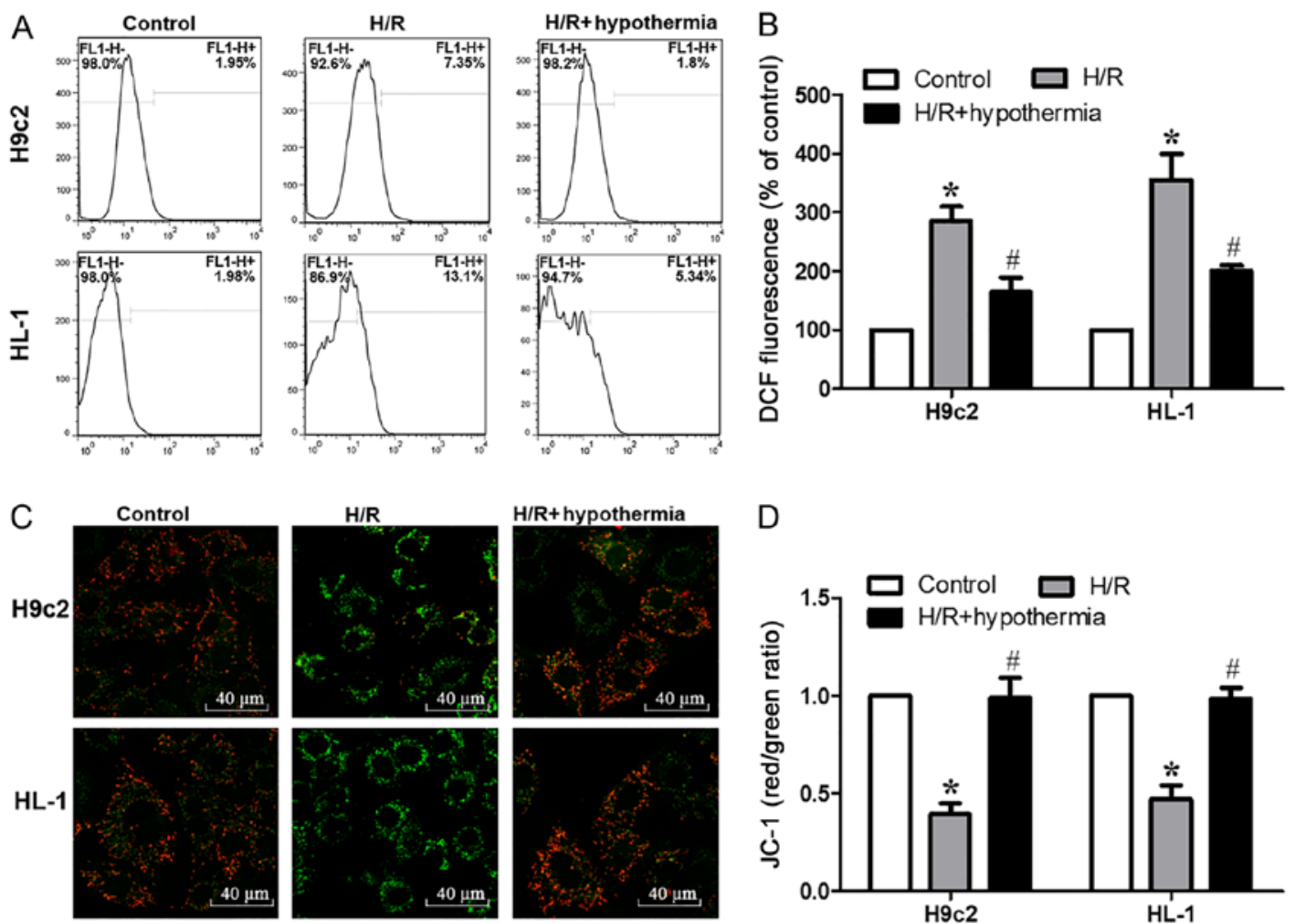

Figure 2. Moderate hypothermia antagonizes the decrease in mitochondrial membrane potential and the production of ROS triggered by H/R in cardiomyocytes. (A) Flow cytometric analysis of ROS generation. Data are presented as the mean \pm SE, $n=5$. (B) Summarized data of DCF fluorescence in HL-1 and H9c2 cells expressed as \% of baseline. (C) Confocal fluorescence images (magnification, x60) of H9c2 and HL-1 cells loaded with JC-1. Scale bar, $40 \mu \mathrm{m}$. (D) Quantified data showing the JC-1 ratio. " $\mathrm{P}<0.05$ vs. control; " $\mathrm{P}<0.05$ vs. H/R. DCF, dichlorofluorescein; H/R, hypoxia/reoxygenation; JC-1, 5,5',6,6'-tetrachloro-1,1',3,3'-tetraethylbenzi-midazolylcarbocyanine iodide; ROS, reactive oxygen species. 

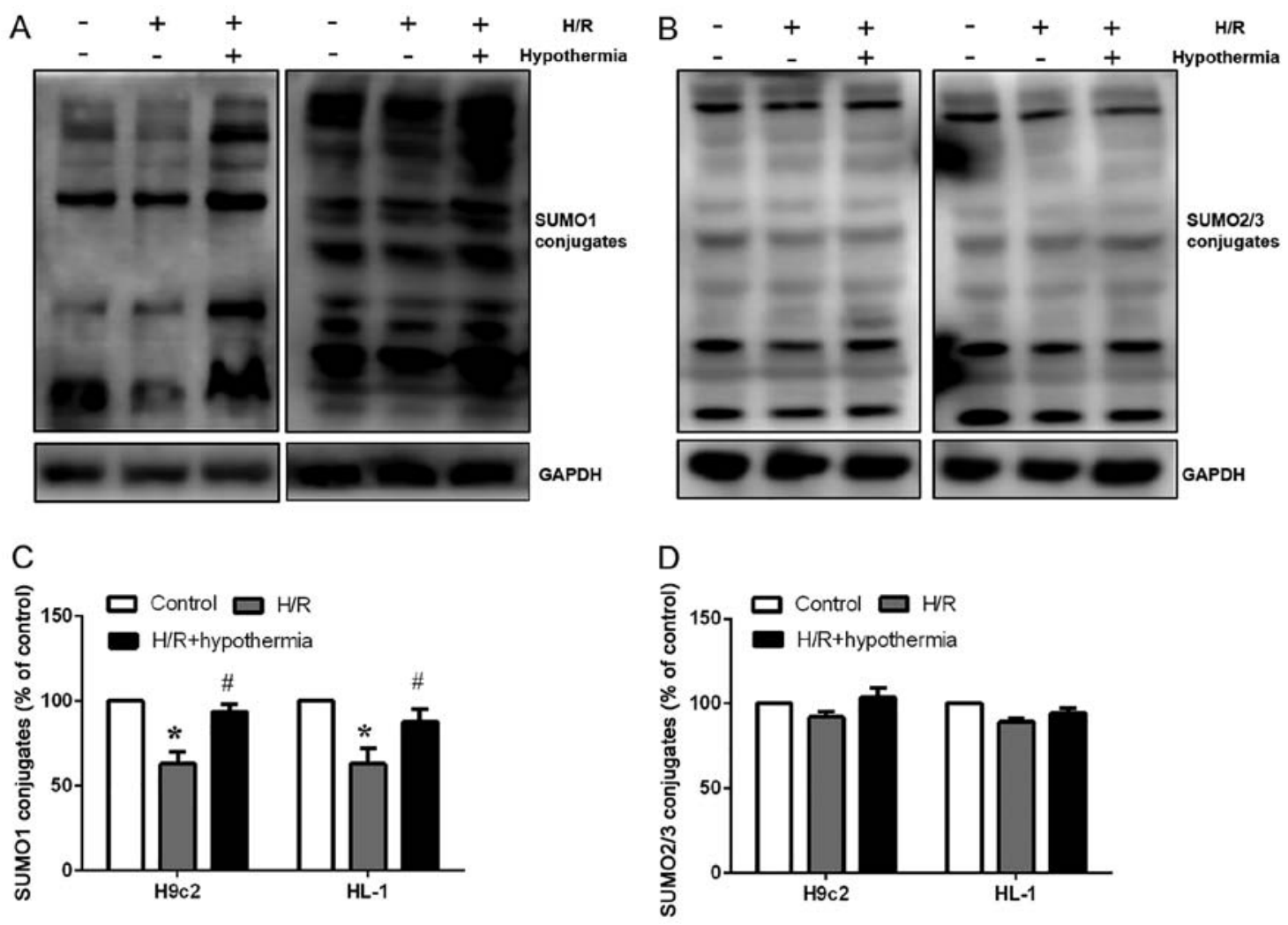

Figure 3. Moderate hypothermia controls SUMOylation of proteins in response to myocardial H/R Injury. (A) SUMO1 expression measured by western blotting. (B) SUMO2/3 expression measured by western blotting. (C) Ratios of SUMO1 conjugate proteins to the control group, $n=6$. (D) Ratios of SUMO2/3 conjugate proteins to the control group $(n=6) .{ }^{*} \mathrm{P}<0.05$ vs. control; ${ }^{\#} \mathrm{P}<0.05 \mathrm{vs}$. H/R. H/R, hypoxia/reoxygenation; SUMO, small ubiquitin-like modifier.

A

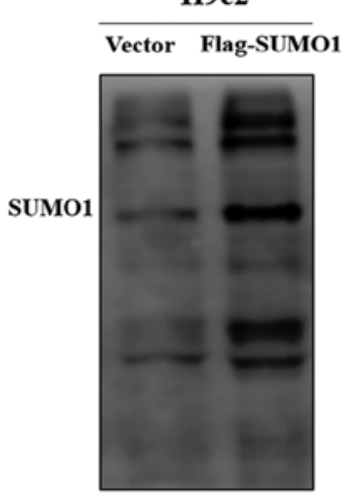

GAPDH
B
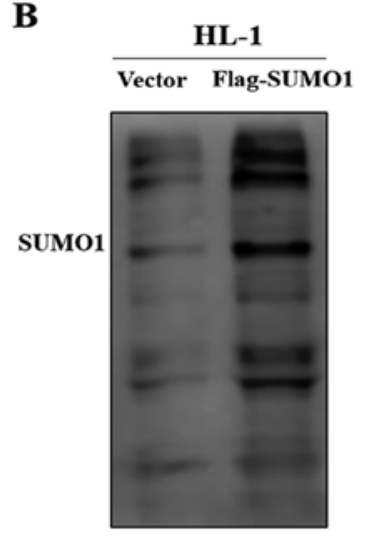

GAPDH
C
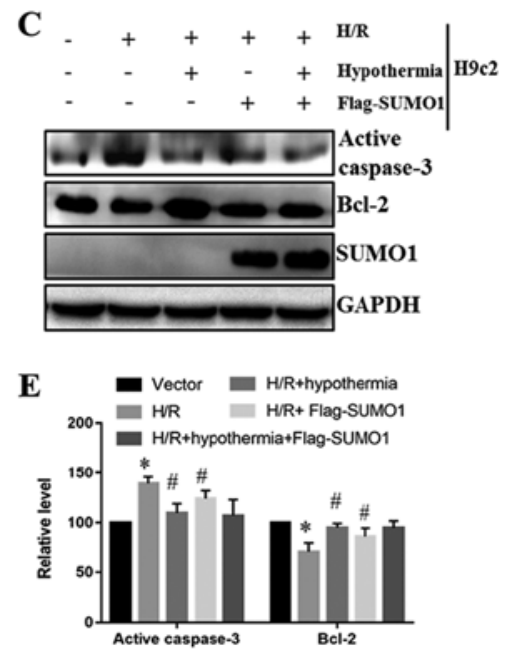

D. ++++ HR

$-\quad+\quad+\quad+$ Hypothermia

- $\quad-\quad+\quad+$ Flag-SUMOI

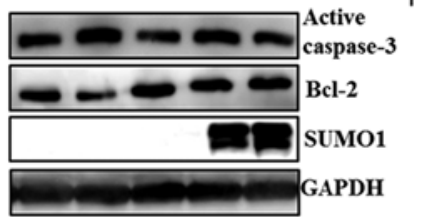

F Vector H/R+hypothermia

F 1 HR HR+Flag-SUMO1

$\left.{ }^{200}\right]$ H/R+hypothermia+Flag-SUMO1

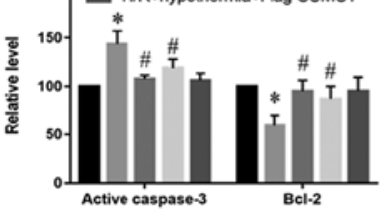

Figure 4. SUMO1 overexpression reversed the effects of H/R on apoptotic proteins. Protein expression levels of SUMO1 in (A) H9c2 cells and (B) HL-1 cells after Flag-SUMO1 transfection under normal conditions. After transfection with Flag-SUMO1, western blotting was used to measure the expression levels of Bcl-2 and caspase-3 in (C) H9c2 and (D) HL-1cells. GAPDH was used to normalize the relative expression levels of caspase-3 and Bcl-2 in (E) H9c2 and (F) HL-1 cells. "P<0.05 vs. Vector; ${ }^{\text {P }}<0.05$ vs. H/R. H/R, hypoxia/reoxygenation; SUMO, small ubiquitin-like modifier.

H/R group (Fig. 3C). However, the protein expression levels of SUMO2/3 were not significantly altered (Fig. 3D). The present results indicated how hypothermia may regulate SUMOylation levels and suggested that, following moderate $\mathrm{TH}$, production of new SUMO1 conjugates may have cytoprotective effects.

Overexpression of SUMO1 strengthens the protective effect of TH on cardiomyocytes under H/R conditions. The present study transfected H9c2 and HL-1 cells with Flag-SUMO1 to induce overexpression of SUMO1 under normal conditions. Western blotting results indicated that SUMO1 overexpression was effective (Fig. 4A and B). Western blotting was also used to determine the protein expression levels of caspase-3 and Bcl-2 in H9c2 (Fig. 4C) and HL-1 (Fig. 4D) cells. The present results suggested that SUMO1 overexpression in the $\mathrm{H} / \mathrm{R}+$ Flag-SUMO1 group reduced the expression levels 
A

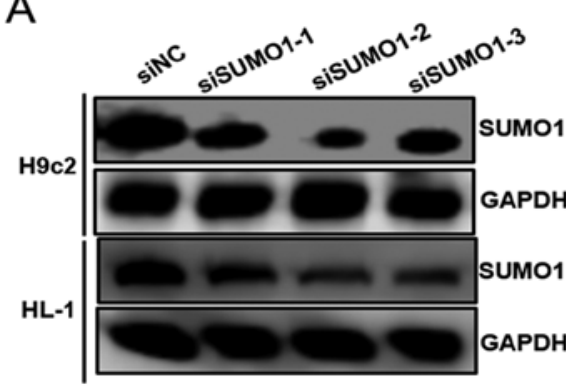

D

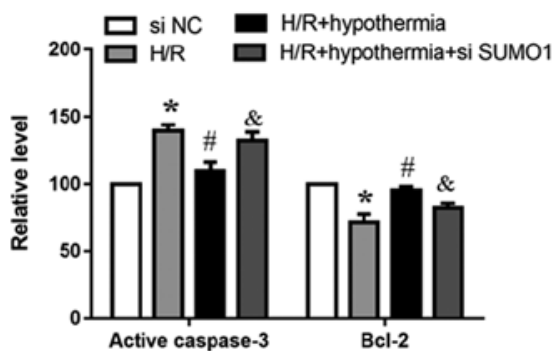

B
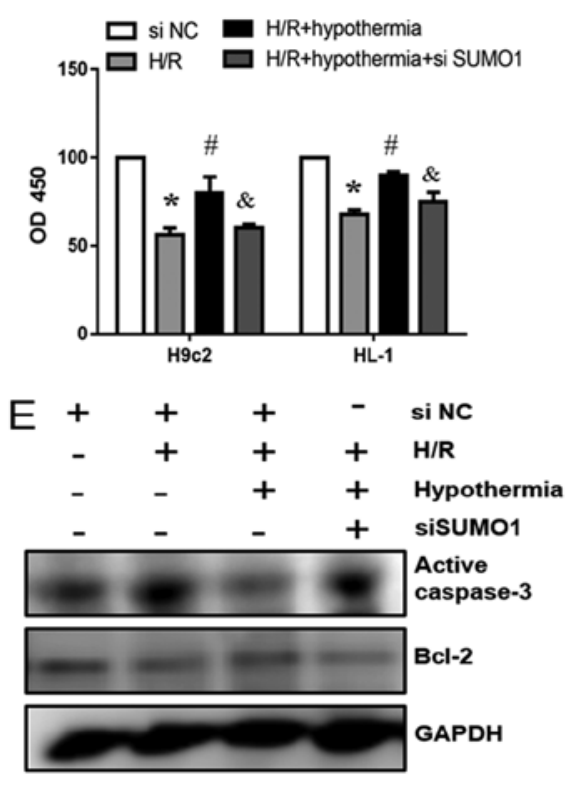

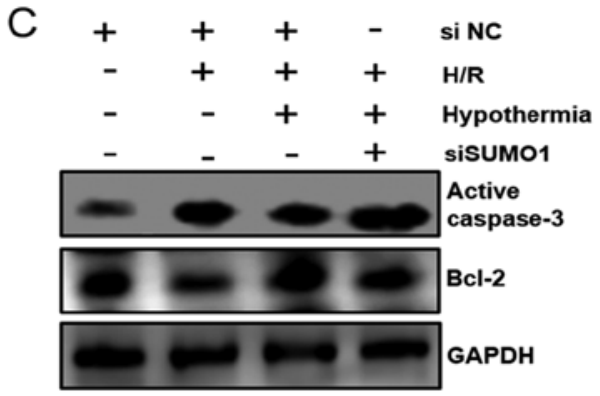

$\mathrm{F}$

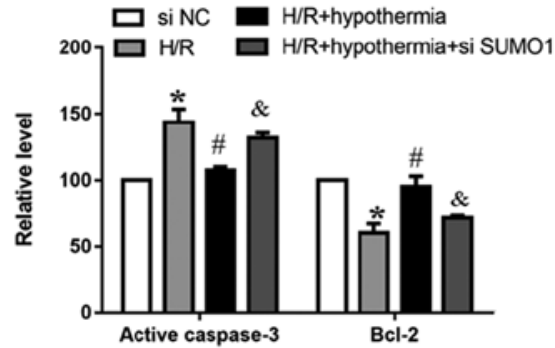

Figure 5. Knockdown of SUMO1 weakens the protective effect of TH on cardiomyocytes. (A) si-SUMO1 transfection caused a marked reduction in SUMO1 protein expression. (B) After transfection with si-SUMO1, a Cell Counting Kit- 8 assay was used to investigate the viability of cells. (C) Western blotting was used to measure the protein expression levels of Bcl-2 and caspase-3 in H9c2 cells. (D) GAPDH was used to determine the relative expression levels of caspase-3 and Bcl-2 in H9c2 cells. (E) Western blotting was used to measure the protein expression levels of Bcl-2 and caspase-3 in HL-1 cells. (F) Relative expression levels of Bcl-2 and caspase-3 normalized to GAPDH in HL-1 cells. ${ }^{*} \mathrm{P}<0.05$ vs. si-NC; ${ }^{\text {P }}<0.05$ vs. H/R; ${ }^{\&} \mathrm{P}<0.05$ vs. H/R $+\mathrm{hypothermia.} \mathrm{H/R,} \mathrm{hypoxia/}$ reoxygenation; NC, negative control; siRNA, small interfering RNA; SUMO, small ubiquitin-like modifier.

of caspase- 3 and increased the expression levels of Bcl-2 compared with the H/R group (Fig. 4E and F). In addition, the similar effect of moderate TH treatment and overexpression of SUMO1 reduced the expression levels of caspase-3 and increased the expression levels of Bcl-2 compared with H/R group of H9c2 and HL-1 cells (Fig. 4E and F). Therefore, the present results suggested that the protective effects of moderate TH may be potentiated through SUMO1 overexpression.

Knockdown of SUMOI weakens the protective effect of TH in cardiomyocytes under $H / R$ conditions. To further investigate whether overexpression of SUMO1 can reduce cardiomyocyte apoptosis, siRNAs (si-SUMO1-1, si-SUMO1-2 and si-SUMO1-3) were used to knock down the expression of SUMO1 in HL-1 and H9c2 cells. Post-transfection with si-SUMO1, the protein expression levels of SUMO1 were decreased compared with in the si-NC groups (Fig. 5A). In addition, cell viability was significantly decreased compared with in the H/R + hypothermia groups (Fig. 5B). As SUMO1 siRNA-2 had the highest efficiency, HL-1 and H9c2 cells were transfected with SUMO1 siRNA-2 and then subjected to $\mathrm{TH}$. Western blotting was performed to assess the protein expression levels of caspase-3 and Bcl-2. In the present study, it was demonstrated that SUMO1 knockdown and hypothermia increased the expression levels of caspase-3 and reduced the expression levels of Bcl-2 compared with in the hypothermia group without siRNA transfection (Fig. 5C-F). Collectively, the present results suggested that SUMO1 affected apoptosis of H9c2 and HL-1 cells following exposure to moderate $\mathrm{TH}$.
Under moderate TH, SUMOI knockdown reduces the mitochondrial membrane potential and enhances ROS production in cardiomyocytes. To further demonstrate that TH antagonizes cardiomyocyte apoptosis by enhancing SUMOylation, SUMO1 was knocked down, and the mitochondrial membrane potential (Fig. 6A) and ROS in cardiomyocytes were measured. It was demonstrated that SUMO1 knockdown significantly increased mitochondrial ROS levels (Fig. 6B) and significantly decreased the mitochondrial membrane potential (Fig. 6C) compared with in the H/R + hypothermia group. Therefore, the present results suggested that SUMO1 knockdown reversed the protective effect of $\mathrm{TH}$ on cardiomyocytes.

\section{Discussion}

While previous studies have demonstrated the importance of applying moderate hypothermia in simulated hypoxic ischemia to attenuate apoptosis of cardiomyocytes, the mechanisms by which moderate hypothermia reduces cell death has not been fully elucidated (29). In the hypoxic ischemia model of HL-1 and $\mathrm{H} 9 \mathrm{c} 2$ cardiomyocytes, the application of hypothermia treatment can effectively maintain the integrity and function of the mitochondrial membrane of cells $(31,32)$. In the present study, it was demonstrated that hypothermia treatment during reoxygenation reduced ROS release and enhanced mitochondrial membrane potential, as indicated by flow cytometric measurement of the intensity of DCF fluorescence and JC-1 detection. Furthermore, these indicators were not significantly different between HL-1 and H9c2 myocardial cells, which is inconsistent with results that HL-1 cells may be more resistant to hypoxia than H9c2 cells (5), possibly because these two 
A
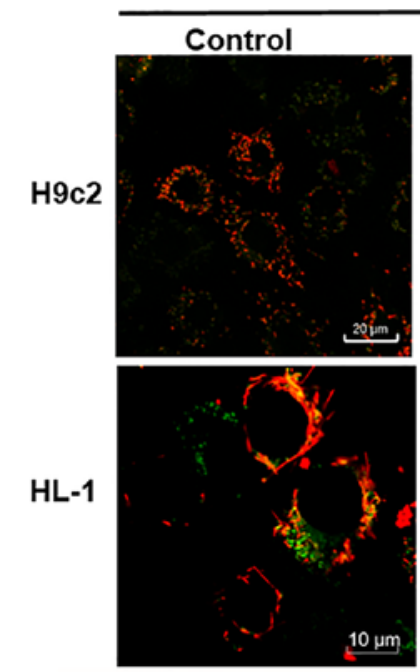

$\mathrm{B}$

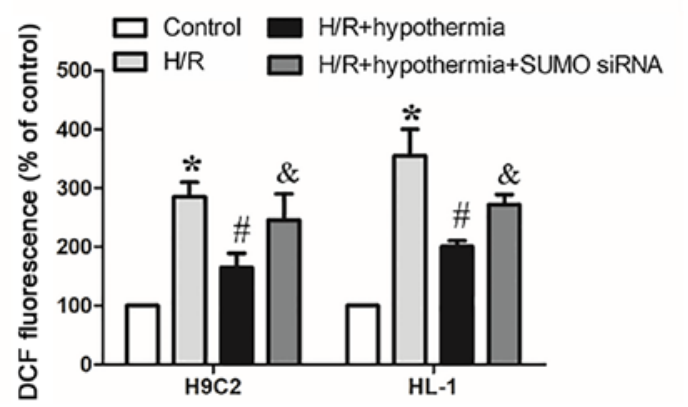

si NC
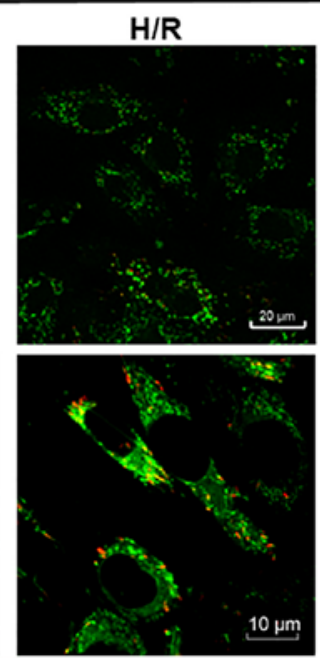

a
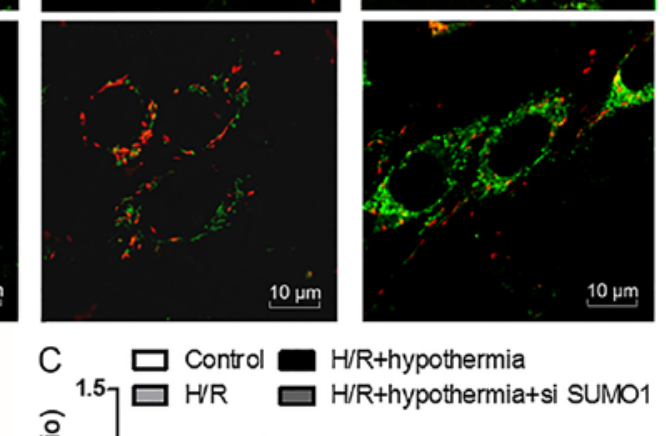
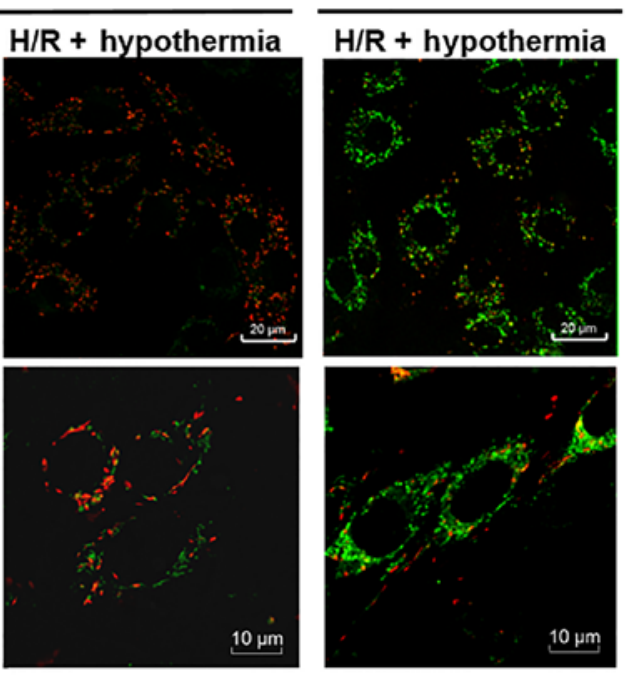

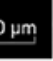

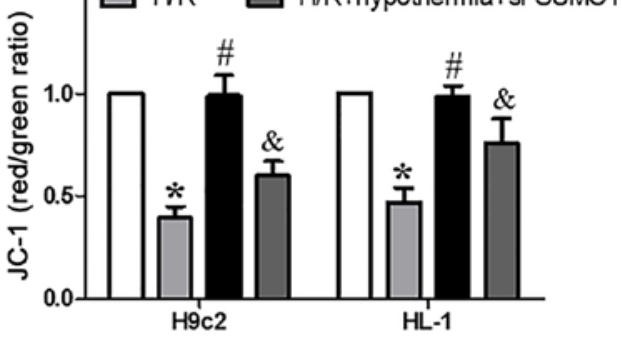

Figure 6. Under moderate hypothermia conditions, SUMO1 knockdown decreases mitochondrial membrane potential and enhances the production of reactive oxygen species in cardiomyocytes. (A) Confocal fluorescence images of HL-1 (magnification, x100) and H9c2 (magnification, x60) cells loaded with JC-1. Scale bars, 20 and $10 \mu \mathrm{m}$. (B) Fluorescence of H9c2 and HL-1 cells loaded with DCF. (C) Quantified data showing the JC-1 ratio. * $\mathrm{P}<0.05$ vs. control (NC siRNA); ${ }^{\#} \mathrm{P}<0.05$ vs. H/R (NC siRNA); ${ }^{\&} \mathrm{P}<0.05$ vs. H/R + hypothermia (NC siRNA). DCF, dichlorofluorescein; H/R, hypoxia/reoxygenation; NC, negative control; JC-1, 5,5',6,6'-tetrachloro-1,1',3,3'-tetraethylbenzi-midazolylcarbocyanine iodide; siRNA, small interfering RNA; SUMO, small ubiquitin-like modifier.

cell lines have a similar tolerance to $\mathrm{H} / \mathrm{R}$ injury. Therefore, the present results support the hypothesis that moderate hypothermia attenuates mitochondrial dysfunction and preserves the integrity of the mitochondrial membrane, which is important for inhibiting apoptosis following $H / R$ injury.

A number of signaling pathways are influenced by SUMOylation (13). SUMOylation plays a role in the progression of several human diseases, such as heart failure and abnormal heart development (16). Previous studies demonstrated that congenital heart defects and retarded cardiac development occurred in mice with SUMO1 knockdown and in mice with SUMO-specific peptidase 2 overexpression $(18,33)$. However, the kinetics of SUMO2/3 and SUMO1 changes during myocardial injury are not fully understood. SUMO1 and SUMO2/3 are coupled by common enzymes and their modification effects on some proteins overlap; these proteins can also specifically target certain proteins and exhibit diverse kinetics $(17,34,35)$. Almost all SUMO1 binds to proteins under physiological conditions (36). Under stress conditions, the dynamic conjugation of SUMO2/3 is increased, which prevents its interaction with target proteins (37). Moreover, in the brain, a strong relationship between SUMO2/3 modifications and neuroprotection has been identified $(38,39)$. In addition, it has been reported that SUMO1 and SUMO2/3 protein modifications are increased during the early stage of myocardial I/R injury (40). However, to the best of our knowledge, no previous study has reported the detailed molecular mechanism of SUMO1 and SUMO2/3 modifications during myocardial H/R-induced injury. In the present study, an increase in SUMO1 protein modification in the early stage of hypothermia was observed when myocardial $\mathrm{H} / \mathrm{R}$ injury was initiated. This is similar to previous studies, in which the effect of SUMO1 on preventing harmful ventricular remodeling and abnormal myocardial cell functional response was reported $(41,42)$. However, no differences were found in SUMO2/3 protein modifications in the present study, which is inconsistent with previous studies that showed an early increase in SUMO2/3 protein expression following myocardial H/R injury (43). The cause of this discrepancy is unclear but may be due to the binding of different types of proteins to SUMOs in different tissues and organs, or due to differences in degrees of binding between target proteins and SUMOs during hypothermia.

It has been reported that $\mathrm{TH}$ as an adjunctive therapy to treat patients with acute myocardial infarction had no significant clinical advantage (44). The hypothesis that moderate hypothermia can stop the apoptosis of cardiomyocytes in response to $\mathrm{H} / \mathrm{R}$-induced injury via multiple pathways has been questioned based on results from conflicting studies. Previous work demonstrated that moderate hypothermia provided cardioprotection after injury triggered by $H / R$ in HL-1 cardiomyocytes by reducing the activation of 
caspase-3 (45). In addition, hypothermia may maintain integrity of the mitochondrial membrane, thus decreasing the release of cytochrome $\mathrm{c}$ and apoptosis-inducing factor, which minimizes caspase-dependent and caspase-independent cell death (29). Bcl-2 proteins largely control mitochondria-associated release of apoptotic factors, but data on the expression of $\mathrm{Bcl}-2$ in response to cryogenic regulation is limited. It was previously reported that moderate hypothermia after ischemia significantly decreased Bax protein expression, and increased Bcl-2 expression levels in a human umbilical vein endothelial cell model (46). In addition, cardiac arrest liquid cooling to $20^{\circ} \mathrm{C}$ for $20 \mathrm{~min}$ in $\mathrm{H} 9 \mathrm{c} 2$ cardiac muscle cells increased Bcl-2 expression levels (31). Moreover, in vitro rabbit heart and in vivo studies have shown that as temperature decreases to $30^{\circ} \mathrm{C}$, the expression levels of $\mathrm{Bcl}-2$ family proteins may increase (47). Thus, the integrity of the mitochondrial membrane may be upheld by increasing Bcl-2 expression levels via TH (32). Apoptosis can be assessed by measuring Bcl-2 expression levels in $\mathrm{H} 9 \mathrm{c} 2$ cardiomyocytes (48). Myocardial energy metabolism is a highly controlled process. The mitochondria forms $40 \%$ of myocardial intracellular volume, and the continuous need for ATP is achieved via aerobic metabolism (49). Therefore, the stability of heart function is highly dependent on normal energy metabolism in the mitochondria. If hypoxic ischemia is succeeded by reoxygenation, reintroduction of nutrients and oxygen contributes to overproduction of ROS, which decreases mitochondrial membrane potential and subsequent apoptosis (5). However, the use of TH as a treatment for patients with severe myocardial infarction has demonstrated beneficial effect in reducing reperfusion injury $(44,50)$. The present study investigated the therapeutic roles of hypothermia in $H / R$, and identified that the expression levels of SUMO1 were increased during hypothermia therapy. The present results suggested that $\mathrm{TH}$ during reperfusion conferred myocardial protection, rather than hypothermia intervention after ischemia and hypoxia.

To further investigate the role of SUMO1, the effects of moderate hypothermia on cell apoptosis, mitochondrial membrane potential and ROS generation were examined. Using a SUMO1 knockdown model, it was demonstrated that SUMO1 protected cardiomyocytes against H/R injury. In addition, ROS generation was decreased and mitochondrial membrane potential was increased during reperfusion as a result of moderate hypothermia treatment. The present study assessed the expression levels of SUMO1 during H/R with moderate hypothermia treatment. It was revealed that SUMO1 knockdown blocked the binding of SUMO1 to its target proteins, which in turn decreased the expression levels of Bcl-2. The present results suggested that the protein expression levels of apoptosis-related caspase-3 were significantly increased. Moreover, siSUMO1 enhanced ROS generation and the mitochondrial membrane potential was decreased. Therefore, the present results suggested that SUMOylation may have a role in the cardioprotective mechanisms of moderate hypothermia. In the present study, overexpression of SUMO1 was sufficient to inhibit the expression of apoptosis-associated proteins, thereby indicating better protection in conjunction with moderate hypothermia. The present results indicated that knockdown of SUMO1 protein expression during moderate hypothermia treatment exacerbated H/R-induced cardiomyocyte injury.
There are still mechanisms that require further investigation; in particular, it is not clear whether moderate hypothermia enhanced the protective effect of myocardial cells via other signaling pathways. A previous study demonstrated that hypothermia reduced the effects of ischemia/reperfusion injury on myocardial cell apoptosis by minimizing autophagy (51). However, the effect of moderate hypothermia on autophagy is a controversial topic, and whether other target proteins (such as the dynamin-related protein-1) bound to SUMO are present in the mitochondria of cardiomyocytes is not fully understood. Due to practical constraints, in the present study the protective mechanisms of hypothermia were studied using in vitro models, which does not accurately simulate the systemic responses to myocardial H/R injury. Moreover, the present study did not conduct a detailed analysis on side effects related to arrhythmia and changes in myocardial contractility. In addition, the present study investigated H/R-induced mitochondrial damage but did not examine alternative sources of myocardial dysfunction after $\mathrm{H} / \mathrm{R}$ injury, including myocardial injury and microvascular obstruction. Götberg et al (8) demonstrated that low temperature after ischemia did not alter the myocardial infarct area of pigs, but did significantly reduce microvascular obstruction. Hence, further research is required to better understand the functional implications of the association between the specific target proteins that conjugate with SUMO1. In vivo models need to be established to investigate the feasibility of moderate hypothermia treatment, while minimizing adverse reactions.

In conclusion, moderate hypothermia significantly enhanced the ability of SUMO1 to bind to target proteins in cardiomyocytes, which suggests an important role in antagonizing myocardial apoptosis and preserving mitochondrial function following hypoxic ischemia/reoxygenation. The present study provides novel evidence on the molecular mechanisms underlying the protective effects of moderate hypothermia. Further research will continue to examine the theoretical mechanisms demonstrated in the present study to facilitate the development of new drugs for improved patient management.

\section{Acknowledgements}

Not applicable.

\section{Funding}

This work was supported by the National Natural Science Foundation of China (grant no. 81471175) and Binhai New Area Health and Family Planning Commission Science and Technology Project (grant nos. 2015BWKY001 and 2014BWKZ006).

\section{Availability of data and materials}

The datasets used and/or analyzed during the current study are available from the corresponding author on reasonable request.

\section{Authors' contributions}

$\mathrm{XL}, \mathrm{YB}$ and $\mathrm{XD}$ conceived and designed the study. JC, XB and LL analyzed the data. JC, XB, YL, XX, CW and YY 
conducted the experiments. JC wrote the manuscript. The final manuscript was read and approved by all the authors.

\section{Ethics approval and consent to participate}

The study protocol was approved by the Ethics Committee of Tianjin Fifth Central Hospital.

\section{Patient consent for publication}

Not applicable.

\section{Competing interests}

The authors declare that they have no competing interests.

\section{References}

1. Nowbar AN, Howard JP, Finegold JA, Asaria P and Francis DP: 2014 global geographic analysis of mortality from ischaemic heart disease by country, age and income: Statistics from World Health Organisation and United Nations. Int J Cardiol 174: 293-298, 2014

2. Joiner ML, Koval OM, Li J, He BJ, Allamargot C, Gao Z, Luczak ED, Hall DD, Fink BD, Chen B, et al: CaMKII determines mitochondrial stress responses in heart. Nature 491: 269-273, 2012

3. Inoue T: Ischemia-reperfusion injury is still a big hurdle to overcome for treatment of acute myocardial infarction. J Cardiol 67: 305-306, 2016.

4. Mandl A, Toth A and Erhardt P: Analysis of apoptosis in isolated primary cardiac myocytes. Methods Mol Biol 559: 293-311, 2009.

5. Kuznetsov AV, Javadov S, Sickinger S, Frotschnig S and Grimm M H9c2 and HL-1 cells demonstrate distinct features of energy metabolism, mitochondrial function and sensitivity to hypoxia-reoxygenation. Biochim Biophys Acta 1853: 276-284, 2015.

6. Azzopardi DV, Strohm B, Edwards AD, Dyet L, Halliday HL, Juszczak E, Kapellou O, Levene M, Marlow N, Porter E, et al; TOBY Study Group: Moderate hypothermia to treat perinatal asphyxial encephalopathy. N Engl J Med 361: 1349-1358, 2009.

7. Bernard S, Buist M, Monteiro O and Smith K: Induced hypothermia using large volume, ice-cold intravenous fluid in comatose survivors of out-of-hospital cardiac arrest: A preliminary report. Resuscitation 56: 9-13,2003.

8. Götberg M, Olivecrona GK, Engblom H, Ugander M, van der Pals J, Heiberg E, Arheden $\mathrm{H}$ and Erlinge D: Rapid short-duration hypothermia with cold saline and endovascular cooling before reperfusion reduces microvascular obstruction and myocardial infarct size. BMC Cardiovase Disord 8: 7, 2008

9. Tissier R, Couvreur N, Ghaleh B, Bruneval P, Lidouren F, Morin D, Zini R, Bize A, Chenoune M, Belair MF, et al: Rapid cooling preserves the ischaemic myocardium against mitochondrial damage and left ventricular dysfunction. Cardiovasc Res 83: 345-353, 2009.

10. Erlinge D, Götberg M, Lang I, Holzer M, Noc M, Clemmensen P, Jensen U, Metzler B, James S, Bötker HE, et al: Rapid endovascular catheter core cooling combined with cold saline as an adjunct to percutaneous coronary intervention for the treatment of acute myocardial infarction. The CHILL-MI trial: A randomized controlled study of the use of central venous catheter core cooling combined with cold saline as an adjunct to percutaneous coronary intervention for the treatment of acute myocardial infarction. J Am Coll Cardiol 63: 1857-1865, 2014.

11. Götberg M, Olivecrona GK, Koul S, Carlsson M, Engblom H Ugander M, van der Pals J, Algotsson L, Arheden H and Erlinge D: A pilot study of rapid cooling by cold saline and endovascular cooling before reperfusion in patients with ST-elevation myocardial infarction. Circ Cardiovasc Interv 3: 400-407, 2010.

12. Zhong N, Kim CY, Rizzu P, Geula C, Porter DR, Pothos EN Squitieri F, Heutink $\mathrm{P}$ and $\mathrm{Xu} \mathrm{J}$ : DJ-1 transcriptionally up-regulates the human tyrosine hydroxylase by inhibiting the sumoylation of pyrimidine tract-binding protein-associated splicing factor. J Biol Chem 281: 20940-20948, 2006.

13. Gareau JR and Lima CD: The SUMO pathway: Emerging mechanisms that shape specificity, conjugation and recognition. Nat Rev Mol Cell Biol 11: 861-871, 2010.
14. Prudden J, Perry JJP, Arvai AS, Tainer JA and Boddy MN: Molecular mimicry of SUMO promotes DNA repair. Nat Struct Mol Biol 16: 509-516, 2009.

15. Wang Z and Prelich G: Quality control of a transcriptional regulator by SUMO-targeted degradation. Mol Cell Biol 29: 1694-1706, 2009.

16. Flotho A and Melchior F: Sumoylation: A regulatory protein modification in health and disease. Annu Rev Biochem 82: 357-385, 2013.

17. Kim EY, Zhang Y, Beketaev I, Segura AM, Yu W, Xi Y, Chang J and Wang J: SENP5, a SUMO isopeptidase, induces apoptosis and cardiomyopathy. J Mol Cell Cardiol 78: 154-164, 2015.

18. Kim EY, Zhang Y, Ye B, Segura AM, Beketaev I, Xi Y, Yu W, Chang J, Li F and Wang J: Involvement of activated SUMO-2 conjugation in cardiomyopathy. Biochim Biophys Acta 1852: 1388-1399, 2015

19. Cai R, Gu J, Sun H, Liu X, Mei W, Qi Y, Xue S, Ren S, Rabinowitz JE, Wang Y, et al: Induction of SENP1 in myocardium contributes to abnormities of mitochondria and cardiomyopathy. J Mol Cell Cardiol 79: 115-122, 2015.

20. Kim EY, Chen L, Ma Y, Yu W, Chang J, Moskowitz IP and Wang J: Enhanced desumoylation in murine hearts by overexpressed SENP2 leads to congenital heart defects and cardiac dysfunction. J Mol Cell Cardiol 52: 638-649, 2012.

21. Montgomery RL, Davis CA, Potthoff MJ, Haberland M, Fielitz J, Qi X, Hill JA, Richardson JA and Olson EN: Histone deacetylases 1 and 2 redundantly regulate cardiac morphogenesis, growth, and contractility. Genes Dev 21: 1790-1802, 2007.

22. Joung H, Kwon S, Kim KH, Lee YG, Shin S, Kwon DH, Lee YU, Kook T, Choe N, Kim JC, et al: Sumoylation of histone deacetylase 1 regulates MyoD signaling during myogenesis. Exp Mol Med 50: e427, 2018.

23. Lee YJ, Mou Y, Klimanis D, Bernstock JD and Hallenbeck JM: Global SUMOylation is a molecular mechanism underlying hypothermia-induced ischemic tolerance. Front Cell Neurosci 8: 416, 2014.

24. Liu X, Ren W, Jiang Z, Su Z, Ma X, Li Y, Jiang R, Zhang J, Yang X: Hypothermia inhibits the proliferation of bone marrow-derived mesenchymal stem cells and increases tolerance to hypoxia by enhancing SUMOylation. Int J Mol Med 40: 1631-1638, 2017.

25. Wei Y and Qing M: G Burkhard M and Wulf P: Deep hypothermia markedly activates the small ubiquitin-like modifier conjugation pathway; implications for the fate of cells exposed to transient deep hypothermic cardiopulmonary bypass. J Cereb Blood Flow Metab (Nihongoban) 29: 886-890, 2009.

26. Nolan JP, Soar J, Cariou A, Cronberg T, Moulaert VR, Deakin CD, Bottiger BW, Friberg H, Sunde K and Sandroni C: European Resuscitation Council and European Society of Intensive Care Medicine Guidelines for Post-resuscitation Care 2015: Section 5 of the European Resuscitation Council Resuscitation Guidelines 2015. Resuscitation 81: 1389-1399, 2015.

27. Lesley C, Yi D, Rita K, Karin P and Sanderson TH: Mitochondrial dynamics: An emerging paradigm in ischemia-reperfusion injury. Curr Pharm Des 19: 6848-6857, 2013.

28. Requião-Moura LR, Durão Junior MS, Matos AC and Pacheco-Silva A: Ischemia and reperfusion injury in renal transplantation: Hemodynamic and immunological paradigms. Einstein (Sao Paulo) 13: 129-135, 2015.

29. Huang CH, Chiang CY, Pen RH, Tsai MS, Chen HW, Hsu CY, Wang TD, Ma MH, Chen SC and Chen WJ: Hypothermia treatment preserves mitochondrial integrity and viability of cardiomyocytes after ischaemic reperfusion injury. Injury 46: 233-239, 2015.

30. Prudent J, Zunino R, Sugiura A, Mattie S, Shore GC and McBride HM: MAPL SUMOylation of Drp1 stabilizes an ER/mitochondrial platform required for cell death. Mol Cell 59: 941-955, 2015.

31. Drescher C, Diestel A, Wollersheim S, Berger F and Schmitt KR: How does hypothermia protect cardiomyocytes during cardioplegic ischemia? Eur J Cardiothorac Surg 40: 352-359, 2011.

32. Krech J, Tong G, Wowro S, Walker C, Rosenthal LM, Berger F and Schmitt KR: Moderate therapeutic hypothermia induces multimodal protective effects in oxygen-glucose deprivation/reperfusion injured cardiomyocytes. Mitochondrion 35: 1-10, 2017.

33. Ashraf H, Pradhan L, Chang EI, Terada R, Ryan NJ, Briggs LE, Chowdhury R,Zárate MA, Sugi Y,Nam HJ, et al: A mouse model of human congenital heart disease: High incidence of diverse cardiac anomalies and ventricular noncompaction produced by heterozygous Nkx2-5 homeodomain missense mutation. Circ Cardiovasc Genet 7: 423-433, 2014. 
34. Iribarren PA, Berazategui MA, Bayona JC, Almeida IC, Cazzulo JJ and Alvarez VE: Different proteomic strategies to identify genuine Small Ubiquitin-like MOdifier targets and their modification sites in Trypanosoma brucei procyclic forms. Cell Microbiol 17: 1413-1422, 2015.

35. Jin LZ, Lu JS and Gao JW: Silencing SUMO2 promotes protection against degradation and apoptosis of nucleus pulposus cells through p53 signaling pathway in intervertebral disc degeneration. Biosci Rep 38: BSR20171523, 2018.

36. Hay RT: SUMO-specific proteases: A twist in the tail. Trends Cell Biol 17: 370-376, 2007.

37. Yang W, Sheng H, Warner DS and Paschen W: Transient global cerebral ischemia induces a massive increase in protein sumoylation. J Cereb Blood Flow Metab 28: 269-279, 2008.

38. Datwyler AL, Lättig-Tünnemann G, Yang W, Paschen W, Lee SL, Dirnagl U, Endres M and Harms C: SUMO2/3 conjugation is an endogenous neuroprotective mechanism. J Cereb Blood Flow Metab 31: 2152-2159, 2011.

39. Li G, Liu X, Su Z and Zhang D: Hypothermia exerts early neuroprotective effects involving protein conjugation of SUMO 2/3 in a rat model of middle cerebral artery occlusion. Mol Med Rep 16: 3217-3223, 2017.

40. Shimizu Y, Lambert JP, Nicholson CK, Kim JJ, Wolfson DW, Cho HC, Husain A, Naqvi N, Chin LS, Li L, et al: DJ-1 protects the heart against ischemia-reperfusion injury by regulating mitochondrial fission. J Mol Cell Cardiol 97: 56-66, 2016.

41. Lee A, Jeong D, Mitsuyama S, Oh JG, Liang L, Ikeda Y, Sadoshima J, Hajjar RJ and Kho C: The role of SUMO-1 in cardiac oxidative stress and hypertrophy. Antioxid Redox Signal 21: 1986-2001, 2014.

42. Shao R, Zhang F-P, Tian F, Friberg PA, Wang X, Sjöland H and Billig H: Increase of SUMO-1 expression in response to hypoxia: Direct interaction with HIF-1 $\alpha$ in adult mouse brain and heart in vivo. FEBS Lett 569: 293-300, 2004.

43. Wang L, Ma Q, Yang W, Mackensen GB and Paschen W: Moderate hypothermia induces marked increase in levels and nuclear accumulation of SUMO2/3-conjugated proteins in neurons. J Neurochem 123: 349-359, 2012.

44. Blatt A, Elbaz-Greener GA, Mizrachi A, J'bara Z, Taraboulos T, Litovchik I, Vered Z and Minha S: Adjunctive mild hypothermia therapy to primary percutaneous coronary intervention in patients with ST segment elevation myocardial infarction complicated with cardiogenic shock: A pilot feasibility study. Cardiol J 22: 285-289, 2015
45. Tong G, Walker C, Bührer C, Berger F, Miera O and Schmitt KR: Moderate hypothermia initiated during oxygen-glucose deprivation preserves HL-1 cardiomyocytes. Cryobiology 70: 101-108, 2015.

46. Yang D, Guo S, Zhang T and Li H: Hypothermia attenuates ischemia/reperfusion-induced endothelial cell apoptosis via alterations in apoptotic pathways and JNK signaling. FEBS Lett 583: 2500-2506, 2009.

47. Ning XH, Chen SH, Xu CS, Li L, Yao LY, Qian K, Krueger JJ, Hyyti OM and Portman MA: Hypothermic protection of the ischemic heart via alterations in apoptotic pathways as assessed by gene array analysis. J Appl Physiol (1985) 92: 2200-2207, 2002.

48. Zhang C, Liu X, Miao J, Wang S, Wu L, Yan D, Li J, Guo W, Wu X and Shen A: Heat shock protein 70 protects cardiomyocytes through suppressing SUMOylation and nucleus translocation of phosphorylated eukaryotic elongation factor 2 during myocardial ischemia and reperfusion. Apoptosis 22: 608-625, 2017.

49. Vogel S, Rath D, Lu J, Chatterjee M, Geisler T and Gawaz M: Elevated mitochondrial membrane potential of circulating monocyte-platelet aggregates in patients with coronary heart disease. Int J Cardiol 181: 135-137, 2015.

50. Mohammad MA, Noc M, Lang I, Holzer M, Clemmensen P, Jensen U, Metzler B and Erlinge D: Proteomics in hypothermia as adjunctive therapy in patients with ST-segment elevation myocardial infarction: A CHILL-MI substudy. Ther Hypothermia Temp Manag 7: 152-161, 2017.

51. Cheng BC, Huang HS, Chao CM, Hsu CC, Chen CY and Chang CP: Hypothermia may attenuate ischemia/reperfusion-induced cardiomyocyte death by reducing autophagy. Int J Cardiol 168: 2064-2069, 2013.

This work is licensed under a Creative Commons Attribution-NonCommercial-NoDerivatives 4.0 International (CC BY-NC-ND 4.0) License. 